\title{
Analytical analysis of fractional-order sequential hybrid system with numerical application
}

\author{
Aziz Khan ${ }^{1} \mathbb{D}$, Zareen A. Khan ${ }^{2 *}$, Thabet Abdeljawad ${ }^{1,3^{*}}$ and Hasib Khan ${ }^{4}$
}

\author{
"Correspondence: \\ zakhan@pnu.edu.sa; \\ tabdeljawad@psu.edu.sa \\ 'Department of Mathematics and \\ Sciences, Prince Sultan University \\ P.O. Box 66833, 11586 Riyadh, Saudi \\ Arabia \\ 2 Department of Mathematical \\ Sciences, College of Science, \\ Princess Nourah bint Abdulrahman \\ University, P.O. Box 84428, Riyadh \\ 11671, Saudi Arabia \\ ${ }^{3}$ Department of Medical Research, \\ China Medical University, Taichung \\ 40402, Taiwan \\ Full list of author information is \\ available at the end of the article
}

\begin{abstract}
We investigate a general sequential hybrid class of fractional differential equations in the Caputo and Atangana-Baleanu fractional senses of derivatives. We consider the existence and uniqueness of solutions and the Hyers-Ulam $(\mathrm{H}-\mathrm{U})$ stability for a general class. We use the Banach and Leray-Schauder alternative theorems for the existence criteria. With the help of nonnegative Green's functions, the fractional-order class is turned into m-equivalent integral forms. As an application of our problem, a fractional-order smoking model in terms of the Atangana-Baleanu derivative is presented as a particular case.
\end{abstract}

\section{Introduction}

Mathematical modeling of dynamical systems and their numerical simulations are widely studied in science and engineering. One of the useful and mostly studied approaches for generalizing the classical models uses the fractional-order operators. The fractional-order operators have a long history from local to nonlocal and from singular to nonsingular kernels. These aspects were recently highlighted in some useful articles. For details, we refer the readers to [1-4].

Fractional-order operators have recently been researched in engineering and science for modeling system dynamics. In the literature, singular and nonsingular kernels are recently well studied. It is difficult to say which one is the greatest right now, but academics always examine several operators for new applications and features. For details, we refer the researchers to [5-7].

The numerical techniques play an important role in the study of dynamical models. For the fractional-order operators, recently, some numerical techniques were developed and applied. For example, the readers can see [8-14]. Using various methodologies, a novel class of mathematical modelings based on hybrid fractional differential equations with hybrid or nonhybrid boundary value conditions has attracted the interest of numerous academics. Nonhomogeneous physical phenomena that occur in their form can be modeled and described using fractional hybrid differential equations. Hybrid differential equations are significant because they incorporate a variety of dynamical systems as special

(c) The Author(s) 2022. This article is licensed under a Creative Commons Attribution 4.0 International License, which permits use sharing, adaptation, distribution and reproduction in any medium or format, as long as you give appropriate credit to the original author(s) and the source, provide a link to the Creative Commons licence, and indicate if changes were made. The images or other third party material in this article are included in the article's Creative Commons licence, unless indicated otherwise in a credit line to the material. If material is not included in the article's Creative Commons licence and your intended use is not permitted by statutory regulation or exceeds the permitted use, you will need to obtain permission directly from the copyright holder. To view a copy of this licence, visit http://creativecommons.org/licenses/by/4.0/. 
instances. The derivative of an unknown function hybrid with nonlinearity is included in this family of differential equations. In addition, hybrid differential equations can be found in several subjects of mathematics and physics, such as the deflection of a curved beam with constant or varying cross-section, a three-layer beam, electromagnetic waves, or gravity-driven flows, and so on. For details, we refer the readers to [15-25].

The general classes of the fractional-order differential equations (FDEs) were considered by experts. This area is still open for sequential fractional differential equations, hybrid FDEs, mixed fractional functional equations, and many more. Dhage [26-28] initiated hybrid FDEs and divided them into two subclasses called the linear and quadratic differential equations. More relevant studies on the hybrid FDEs can be found in [29-36]. In this paper, we present a system of hybrid sequential FDEs with two different fractional operators, the Caputo and Atangana-Baleanu operators. Our presumed system of hybrid sequential FDEs with initial and boundary conditions is

$$
\begin{aligned}
& { }^{c} \mathcal{D}^{\alpha_{i}}\left[{ }^{A B C} \mathcal{D}^{e_{i}} u_{i}(t)+\sum_{1}^{m} \mathcal{F}_{i}\left(t, u_{i}(t)\right)\right]=-\lambda_{i}\left(t, u_{i}(t)\right), \quad t \in \mathrm{I}=[0,1], \\
& u_{i}(0)=0, \quad u_{i}^{*}(1)=\Delta_{i},\left.\quad \mathcal{F}_{i}\left(t, u_{i}(t)\right)\right|_{t=0}=0,
\end{aligned}
$$

where $\Delta_{i}=\left.\frac{1-\varrho_{i}}{\mathcal{B}\left(\varrho_{i}\right)}\left(\sum_{i=1}^{m} \mathcal{F}_{i}\left(t, u_{i}(t)\right)+\mathcal{I}^{\alpha_{i}} \lambda_{i}\left(t, u_{i}^{*}(t)\right)\right)\right|_{t=1}, 0<\alpha_{i} \leq 1,0 \leq \varrho_{i} \leq 1$, the functions $u_{i}: \mathrm{I} \rightarrow \mathcal{R}_{e}, i=1,2, \ldots, m$, are continuous, and $\lambda_{i}, \mathcal{F}_{i}: \mathrm{I} \times \mathcal{R}_{e} \rightarrow \mathcal{R}_{e}, h_{i}: \mathrm{I} \times \mathcal{R}_{e} \rightarrow \mathcal{R}_{e}$ $(i=1,2, \ldots, m)$ satisfy the Carathéodory conditions and are continuous functions. The ${ }^{c} \mathcal{D}^{\alpha_{i}}$ are in the Caputo sense, whereas ${ }^{A B C} \mathcal{D}^{e_{i}}, i=1,2, \ldots, m$, are in the ABC-sense of fractional derivatives. This sort of general sequential hybrid problems have not been studied in the literature. To know whether such problems can have solutions and applications, we consider the existence, uniqueness, stability, and applications in the dynamical systems. Dhage [26-28] and the references therein give more information to the readers on the theory and applications of the problem. We use the fixed point approach for the theoretical analysis and Euler discretization technique in application aspects. For details of the nonlinear models and their simulations, we refer the readers to [37-54].

\subsection{Basic definitions of fractional calculus}

The concept of a nonsingular kernel was given by Caputo and Fabrizio [55] by replacing the singular kernel by exponential function. This work was then studied for some essential properties in [56]. Later on, Atangana and Baleanu [57] modified the concept of a nonsingular kernel with the replacement of the exponential kernel by the Mittag-Lefflerkernel. They called the new fractional-order differential operator the Atangana-Baleanu fractional derivative, which was recently well studied by many authors. We refer the readers to some related work on these operators and its applications in [55-57] and the references therein.

Definition 1.1 ([57]) The ABC-fractional differential operator on $\psi \in H^{1}(a, b), b>a$, for $\varrho_{1} \in[0,1]$ is

$$
{ }_{a}^{A B C}{ }_{a} \mathcal{D}_{\tau}^{\varrho_{1}} \psi(\tau)=\frac{B\left(\varrho_{1}\right)}{1-\varrho_{1}} \int_{a}^{\tau} \psi^{\prime}(s) E_{\varrho_{1}}\left[\frac{-\varrho_{1}(\tau-s)_{1}^{\varrho}}{1-\varrho_{1}}\right] d s,
$$


where $B\left(\varrho_{1}\right)$ is a normalizer function satisfying $B(0)=B(1)=1$. If the function does not belong to $H^{1}(a, b), b>a$, then the fractional-order derivative of order $\varrho_{1} \in[0,1]$ has the form

$$
{ }_{a}^{A B C} \mathcal{D}_{\tau}^{\varrho_{1}} \psi(\tau)=\frac{\varrho_{1} B\left(\varrho_{1}\right)}{1-\varrho_{1}} \int_{a}^{\tau}(\psi(\tau)-\psi(s)) E_{\varrho_{1}}\left[\frac{-\varrho_{1}(\tau-s)^{\varrho_{1}}}{1-\varrho_{1}}\right] d s,
$$

where $H^{1}(a, b)$ is the set of functions with continuous first derivatives.

Definition 1.2 ([57]) For $\psi \in H^{1}(a, b), b>a, \varrho_{1} \in[0,1]$, the ABR-fractional derivative is

$$
{ }_{A B R}{ }_{a} \mathcal{D}_{\tau}^{\varrho_{1}} \psi(\tau)=\frac{B\left(\varrho_{1}\right)}{1-\varrho_{1}} \frac{d}{d \tau} \int_{a}^{\tau} \psi(s) E_{\varrho_{1}}\left[\frac{-\varrho_{1}(\tau-s)^{\varrho_{1}}}{1-\varrho_{1}}\right] d s .
$$

Definition 1.3 ([57]) The AB-integral of $\psi \in H^{1}(a, b), b>a, 0<\varrho_{1}<1$, is given by

$$
{ }_{a}^{A B} \mathcal{I}_{\tau}^{\varrho_{1}} \psi(\tau)=\frac{1-\varrho_{1}}{B\left(\varrho_{1}\right)} \psi(\tau)+\frac{\varrho_{1}}{B\left(\varrho_{1}\right) \Gamma\left(\varrho_{1}\right)} \int_{a}^{\tau} \psi(s)(\tau-s)^{\varrho_{1}-1} d s .
$$

The $\mathrm{ABC}$ and $\mathrm{ABR}$ are related to each other by the following relation.

Theorem 1.4 ([57]) Let $\psi \in H^{1}(a, b), b>a$. Then for a fractional-order $\varrho_{1} \in[0,1]$, we have

$$
{ }_{0}^{A B C} \mathcal{D}_{t}^{\varrho_{1}} \psi(t)={ }_{0}^{A B R} \mathcal{D}_{t}^{\varrho_{1}} \psi(t)+H(t) .
$$

In our results, we will need to the following result.

Lemma 1.5 ([58]) The AB fractional derivative and AB fractional integral of the function $\psi$ satisfy the Newton-Leibniz formula

$$
{ }_{a}^{A B} \mathcal{I}_{\tau}^{Q_{1}}\left({ }^{A B C}{ }_{a} \mathcal{D}_{\tau}^{Q_{1}} \psi(\tau)\right)=\psi(\tau)-\psi(a) .
$$

\section{Existence of solution}

In this section, we obtain the existence of a solution for the suggested hybrid FDE (1) with the help of fixed point technique.

Lemma 2.1 The solution of the sequential hybrid system (1) is

$$
\begin{aligned}
u_{i}= & -\frac{1-\varrho_{i}}{\mathcal{B}\left(\varrho_{i}\right)}\left(\sum_{1}^{m} \mathcal{F}_{i}\left(t, u_{i}(t)\right)+\mathcal{I}^{\alpha_{i}} \lambda_{i}\left(t, u_{i}(t)\right)\right) \\
& +\mathcal{B}\left(\varrho_{i}\right)\left(\frac{1}{\Gamma\left(\varrho_{i}\right)} \int_{0}^{1} \mathcal{G}_{i}(t, s) \sum_{1}^{m} \mathcal{F}_{i}\left(s, u_{i}(s)\right) d s\right. \\
& \left.+\frac{1}{\Gamma\left(\varrho_{i}+\alpha_{i}\right)} \int_{0}^{1} \mathcal{H}_{i}(t, s) \lambda_{i}\left(s, u_{i}(s)\right) d s\right),
\end{aligned}
$$

where

$$
\mathcal{G}_{i}(t, s)=\frac{1}{\Gamma\left(\varrho_{i}\right)} \begin{cases}(1-s)^{\varrho_{i}-1}, & t \leq s \\ (1-s)^{\varrho_{i}-1}-(t-s)^{\varrho_{i}-1}, & t \geq s\end{cases}
$$




$$
\mathcal{H}_{i}(t, s)=\frac{1}{\Gamma\left(\varrho_{i}+\alpha_{i}\right)} \begin{cases}(1-s)^{\varrho_{i}+\alpha_{i}-1}, & t \leq s \\ (1-s)^{\varrho_{i}+\alpha_{i}-1}-(t-s)^{\varrho_{i}+\alpha_{i}-1}, & t \geq s\end{cases}
$$

for $i=1,2, \ldots, m$.

Proof Applying $\left(I^{\alpha_{i}}\right)$ to system (1), we have

$$
{ }^{A B C} \mathcal{D}^{\varrho_{i}} u_{i}(t)+\sum_{i=1}^{m} \mathcal{F}_{i}\left(t, u_{i}(t)\right)=-I^{\alpha_{i}} \lambda_{i}\left(t, u_{i}(t)\right)+C_{1, i}
$$

for $i=1,2, \ldots, m$. Using the initial conditions $u_{i}(0)=0$, for $i=1,2, \ldots, m$, we have $C_{1, i}=0$. This implies

$$
{ }^{A B C} \mathcal{D}^{\varrho_{i}} u_{i}(t)=-\mathcal{I}^{\alpha_{i}} \lambda_{i}\left(t, u_{i}(t)\right)-\sum_{i=1}^{m} \mathcal{F}_{i}\left(t, u_{i}(t)\right) \quad \text { for } i=1,2, \ldots, m
$$

With the help of ABC-fractional calculus and (12) we have

$$
\begin{aligned}
u_{i}(t)= & -\frac{1-\varrho_{i}}{\mathcal{B}\left(\varrho_{i}\right)}\left(\mathcal{I}^{\alpha_{i}} \lambda_{i}\left(t, u_{i}(t)\right)+\sum_{1}^{m} \mathcal{F}_{i}\left(t, u_{i}(t)\right)\right) \\
& -\mathcal{B}\left(\varrho_{i}\right) \mathcal{I}^{\varrho}\left(\sum_{1}^{m} \mathcal{F}_{i}\left(t, u_{i}(t)\right)+\mathcal{I}^{\alpha_{i}} \lambda_{i}\left(t, u_{i}(t)\right)\right)+C_{2, i} .
\end{aligned}
$$

Since of $u_{i}(1)=0$, by (13) we have $C_{2, i}=\left.\mathcal{B}\left(\varrho_{i}\right) I^{\varrho_{i}}\left(\sum_{1}^{m} \mathcal{F}_{i}\left(t, u_{i}(t)\right)+I^{\alpha_{i}+\varrho_{i}} \lambda_{i}\left(t, u_{i}(t)\right)\right)\right|_{t=1}$ for $i=1,2, \ldots, m$. This leads to the following equivalent integral system:

$$
\begin{aligned}
u_{i}(t)= & -\frac{1-\varrho_{i}}{\mathcal{B}\left(\varrho_{i}\right)}\left(\sum_{1}^{m} \mathcal{F}_{i}\left(t, u_{i}(t)\right)+\mathcal{I}^{\alpha_{i}} \lambda_{i}\left(t, u_{i}(t)\right)\right) \\
& -\mathcal{B}\left(\varrho_{i}\right) \mathcal{I}^{\varrho_{i}}\left(\sum_{1}^{m} \mathcal{F}_{i}\left(t, u_{i}(t)\right)+\mathcal{I}^{\alpha_{i}} \lambda_{i}\left(t, u_{i}(t)\right)\right) \\
& +\left.\mathcal{B}\left(\varrho_{i}\right) I^{\varrho}\left(\sum_{1}^{m} \mathcal{F}_{i}\left(t, u_{i}(t)\right)+I^{\alpha_{i}} \lambda_{i}\left(t, u_{i}(t)\right)\right)\right|_{t=1} \\
= & -\frac{1-\varrho_{i}}{\mathcal{B}\left(\varrho_{i}\right)}\left(\sum_{1}^{m} \mathcal{F}_{i}\left(t, u_{i}(t)\right)+\mathcal{I}^{\alpha_{i}} \lambda_{i}\left(t, u_{i}(t)\right)\right) \\
& +\mathcal{B}\left(\varrho_{i}\right)\left(\left({ }_{0} I_{1}^{\varrho_{i}+\alpha_{i}}-{ }_{0} I_{t}^{\varrho_{i}+\alpha_{i}}\right) \lambda_{i}\left(t, u_{i}(t)\right)+\left({ }_{0} I_{1}^{\varrho_{i}}-{ }_{0} I_{t}^{\varrho_{i}}\right) \sum_{1}^{m} \mathcal{F}_{i}\left(t, u_{i}(t)\right)\right) \\
= & \mathcal{B}\left(\varrho_{i}\right)\left(\frac{1}{\Gamma\left(\varrho_{i}\right)} \int_{0}^{1}\left((1-s)^{\varrho_{i}-1}-(t-s)^{\varrho_{i}-1}\right) \sum_{1}^{m} \mathcal{F}_{i}\left(s, u_{i}(s)\right) d s\right. \\
& \left.+\frac{1}{\Gamma\left(\varrho_{i}+\alpha_{i}\right)} \int_{0}^{1}\left((1-s)^{\varrho_{i}-1+\alpha_{i}}-(t-s)^{\varrho_{i}-1+\alpha_{i}}\right) \lambda_{i}\left(s, u_{i}(s)\right) d s\right) \\
& -\frac{\varrho_{i}}{\mathcal{B}\left(\varrho_{i}\right)}\left(\sum_{1}^{m} \mathcal{F}_{i}\left(t, u_{i}(t)\right)+\mathcal{I}^{\alpha_{i}} \lambda_{i}\left(t, u_{i}(t)\right)\right) \\
&
\end{aligned}
$$




$$
\begin{aligned}
= & -\frac{1-\varrho_{i}}{\mathcal{B}\left(\varrho_{i}\right)}\left(\sum_{1}^{m} \mathcal{F}_{i}\left(t, u_{i}(t)\right)+\mathcal{I}^{\alpha_{i}} \lambda_{i}\left(t, u_{i}(t)\right)\right) \\
& +\mathcal{B}\left(\varrho_{i}\right)\left(\frac{1}{\Gamma\left(\varrho_{i}\right)} \int_{0}^{1} \mathcal{G}_{i}(t, s) \sum_{1}^{m} \mathcal{F}_{i}\left(s, u_{i}(s)\right) d s\right. \\
& \left.+\frac{1}{\Gamma\left(\varrho_{i}+\alpha_{i}\right)} \int_{0}^{1} \mathcal{H}_{i}(t, s) \lambda_{i}\left(s, u_{i}(s)\right) d s\right)
\end{aligned}
$$

for $i=1,2, \ldots, m$. The $\mathcal{G}_{i}(t, s)$ and $\mathcal{H}_{i}(t, s), i=1,2, \ldots, m$, are defined in (9) and (10), respectively.

In this paper, we consider the Banach space $\mathcal{B}=\left\{u_{i}(t): u_{i}(t) \in \mathcal{C}\left([0,1], \mathcal{R}_{e}\right)\right.$ for $\left.t \in[0,1]\right\}$ with norm $\left\|u_{i}\right\|=\max _{t \in[0,1]}\left|u_{i}(t)\right|, i=1,2, \ldots, m$.

Let $\mathcal{T}_{i}: \mathcal{C}\left([0,1], \mathcal{R}_{e}\right) \rightarrow \mathcal{C}([0,1], \mathbb{R}), i=1,2, \ldots, m$, be the operators defined by

$$
\begin{aligned}
\mathcal{T}_{i} u_{i}(t)= & -\frac{1-\varrho_{i}}{\mathcal{B}\left(\varrho_{i}\right)}\left(\sum_{1}^{m} \mathcal{F}_{i}\left(t, u_{i}(t)\right)+\mathcal{I}^{\alpha_{i}} \lambda_{i}\left(t, u_{i}(t)\right)\right) \\
& +\mathcal{B}\left(\varrho_{i}\right)\left(\frac{1}{\Gamma\left(\varrho_{i}\right)} \int_{0}^{1} \mathcal{G}_{i}(t, s) \sum_{i=1}^{m} \mathcal{F}_{i}\left(s, u_{i}(s)\right) d s\right. \\
& \left.+\frac{1}{\Gamma\left(\alpha_{i}+\varrho_{i}\right)} \int_{0}^{1} \mathcal{H}_{i}(t, s) \lambda_{i}\left(s, u_{i}(s)\right) d s\right) .
\end{aligned}
$$

The $\mathcal{G}_{i}(t, s)$ and $\mathcal{H}_{i}(t, s), i=1,2, \ldots, m$, are given in (9) and (10), respectively. By (15) the fixed points of the operators $\mathcal{T}_{i}$ give the solutions of the hybrid system (1). By (9) and (10) the functions $\mathcal{G}_{i}(s, t)$ and $\mathcal{H}_{i}(s, t)$ are clearly positive operators for both $t \leq s$ and $t \geq s$, for $t, s \in(0,1]$ and $i=1,2, \ldots, m$.

Lemma 2.2 Assume that for some $\zeta_{i}^{1}, \zeta_{i}^{2} \in \mathcal{R}_{e}$ and $u_{i}, u_{i}^{*} \in \mathcal{C}, t \in[0, k]$, we have

$$
\begin{aligned}
& \left|\lambda_{i}\left(t, u_{i}\right)-\lambda_{i}\left(t, \bar{u}_{i}\right)\right| \leq \zeta_{i}^{1}\left|u_{i}-\bar{u}_{i}\right|, \\
& \left|\mathcal{F}_{i}\left(t, u_{i}\right)-\mathcal{F}_{i}\left(t, \bar{u}_{i}\right)\right| \leq \zeta_{i}^{2}\left|u_{i}-\bar{u}_{i}\right|,
\end{aligned}
$$

and

$$
\begin{aligned}
\eta_{i}= & \frac{1-\varrho_{i}}{\mathcal{B}\left(\varrho_{i}\right)}\left(m\left(\zeta_{i}^{2} \eta_{i}+\wp_{2}\right)+\frac{1}{\Gamma\left(\alpha_{i}+1\right)}\left(\zeta_{i}^{1} \eta_{i}+\wp_{1}\right)\right) \\
& +\mathcal{B}\left(\varrho_{i}\right)\left(\frac{1}{\Gamma\left(\varrho_{i}\right)} \frac{2 k^{\varrho_{i}}}{\Gamma(\varrho+1)} m\left(\zeta_{i}^{2} \eta_{i}+\wp_{2}\right)\right. \\
& \left.+\frac{1}{\Gamma\left(\varrho_{i}+\alpha_{i}\right)} \frac{2 k^{\varrho_{i}+\alpha_{i}}}{\Gamma\left(\varrho+\alpha_{i}+1\right)}\left(\zeta_{i}^{1} \eta_{i}+\wp_{1}\right)\right)
\end{aligned}
$$

for $\eta_{i}<1, i=1,2, \ldots, m$. Then (1) has a unique solution.

Proof In this proof, the subscripts $i=1,2, \ldots, n$. Assume that $\sup _{t \in[0, k]}\left|\lambda_{i}(t, 0)\right|=\wp_{1}<\infty$, $\sup _{t \in[0, k]}\left|\mathcal{F}_{i}(t, 0)\right|=\wp_{2}<\infty, \mathcal{S}_{\eta_{i}}=\left\{u_{i} \in \mathcal{C}\left([0, k], \mathcal{R}_{e}\right):\left\|u_{i}\right\|<\eta_{i}\right\}$, and $k \geq 1$. For $u_{i} \in \mathcal{S}_{\eta_{i}}$ and 
$t \in[0, k]$, we have

$$
\begin{aligned}
\left|\lambda_{i}\left(t, u_{i}(t)\right)\right| & =\left|\lambda_{i}\left(t, u_{i}(t)\right)-\lambda_{i}(t, 0)+\lambda_{i}(t, 0)\right| \\
& \leq\left|\lambda_{i}\left(t, u_{i}(t)\right)-\lambda_{i}(t, 0)\right|+\left|\lambda_{i}(t, 0)\right| \\
& \leq \zeta_{i}^{1}\left|u_{i}(t)\right|+\left|\lambda_{i}(t, 0)\right| \\
& \leq \zeta_{i}^{1} \eta_{i}+\wp_{1} .
\end{aligned}
$$

Similarly, for $v_{i} \in \mathcal{S}_{\eta_{i}}$ and $t \in[0, k]$, we have

$$
\begin{aligned}
\left|\mathcal{F}_{i}\left(t, v_{i}(t)\right)\right| & =\left|\mathcal{F}_{i}\left(t, v_{i}(t)\right)-\mathcal{F}_{i}(t, 0)+\mathcal{F}_{i}(t, 0)\right| \\
& \leq\left|\mathcal{F}_{i}\left(t, v_{i}(t)\right)-\mathcal{F}_{i}(t, 0)\right|+\left|\mathcal{F}_{i}(t, 0)\right| \\
& \leq \zeta_{i}^{2}\left|v_{i}(t)\right|+\left|\mathcal{F}_{i}(t, 0)\right| \\
& \leq \zeta_{i}^{2} \eta_{i}+\wp_{2} .
\end{aligned}
$$

Furthermore, for $t \geq s$, by (9) we have

$$
\begin{aligned}
\int_{0}^{1}\left|\mathcal{G}_{i}(t, s)\right| d s & =\frac{1}{\Gamma(\varrho)} \int_{0}^{1}\left|(1-s)^{\varrho_{i}-1}-(t-s)^{\varrho_{i}-1}\right| d s \\
& \leq \frac{\left((1-s)^{\varrho_{i}}+(t-s)^{\varrho_{i}}\right)}{\Gamma(\varrho+1)} \leq \frac{2 k^{\varrho_{i}}}{\Gamma(\varrho+1)},
\end{aligned}
$$

and for $t \leq s$, we have

$$
\int_{0}^{1}\left|\mathcal{G}_{i}(t, s)\right| d s=\frac{1}{\Gamma(\varrho)} \int_{0}^{1}\left|(1-s)^{\varrho_{i}-1}\right| d s \leq \frac{k^{\varrho_{i}}}{\Gamma(\varrho+1)} .
$$

Now, consider the Green's functions $\mathcal{H}_{i}(t, s)$ given by (10). For the case $t \geq s$, we have

$$
\begin{aligned}
\int_{0}^{1}\left|\mathcal{H}_{i}(t, s)\right| d s & =\frac{1}{\Gamma\left(\varrho+\alpha_{i}\right)} \int_{0}^{1}\left|(1-s)^{\varrho_{i}+\alpha_{i}-1}-(t-s)^{\varrho_{i}+\alpha_{i}-1}\right| d s \\
& \leq \frac{1}{\Gamma\left(\varrho+\alpha_{i}+1\right)}\left((1-s)^{\varrho_{i}+\alpha_{i}}+(t-s)^{\varrho_{i}+\alpha_{i}}\right) \leq \frac{2 k^{\varrho_{i}+\alpha_{i}}}{\Gamma\left(\varrho+\alpha_{i}+1\right)}
\end{aligned}
$$

and for $t \leq s$, we have

$$
\int_{0}^{1}\left|\mathcal{H}_{i}(t, s)\right| d s=\frac{1}{\Gamma\left(\alpha_{i}+\varrho\right)} \int_{0}^{1}\left|(1-s)^{\varrho_{i}-1+\alpha_{i}}\right| d s \leq \frac{k^{\alpha_{i}+\varrho_{i}}}{\Gamma\left(\alpha_{i}+\varrho+1\right)} .
$$

With the help of (15), for $t \geq s$, we have

$$
\begin{aligned}
\left|\mathcal{T}_{i} u_{i}(t)\right|=\mid & -\frac{1-\varrho_{i}}{\mathcal{B}\left(\varrho_{i}\right)}\left(\sum_{1}^{m} \mathcal{F}_{i}\left(t, u_{i}(t)\right)+\mathcal{I}^{\alpha_{i}} \lambda_{i}\left(t, u_{i}(t)\right)\right) \\
& +\mathcal{B}\left(\varrho_{i}\right)\left(\frac{1}{\Gamma\left(\varrho_{i}+\alpha_{i}\right)} \int_{0}^{1} \mathcal{H}_{i}(t, s) \lambda_{i}\left(s, u_{i}(s)\right) d s\right.
\end{aligned}
$$




$$
\begin{aligned}
& \left.+\frac{1}{\Gamma\left(\varrho_{i}\right)} \int_{0}^{1} \mathcal{G}_{i}(t, s) \sum_{1}^{m} \mathcal{F}_{i}\left(s, u_{i}(s)\right) d s\right) \mid \\
\leq & \frac{1-\varrho_{i}}{\mathcal{B}\left(\varrho_{i}\right)}\left(m\left(\zeta_{i}^{2} \eta_{i}+\wp_{2}\right)+\frac{1}{\Gamma\left(\alpha_{i}+1\right)}\left(\zeta_{i}^{1} \eta_{i}+\wp_{1}\right)\right) \\
& +\mathcal{B}\left(\varrho_{i}\right)\left(\frac{1}{\Gamma\left(\varrho_{i}\right)} \int_{0}^{1} \mathcal{G}_{i}(t, s) m\left(\left(\zeta_{i}^{2} \eta_{i}+\wp_{2}\right)\right) d s\right. \\
& \left.+\frac{1}{\Gamma\left(\varrho_{i}+\alpha_{i}\right)} \int_{0}^{1} \mathcal{H}_{i}(t, s)\left(\left(\zeta_{i}^{1} \eta_{i}+\wp_{1}\right)\right) d s\right) \\
\leq & \frac{1-\varrho_{i}}{\mathcal{B}\left(\varrho_{i}\right)}\left(m\left(\zeta_{i}^{2} \eta_{i}+\wp_{2}\right)+\frac{1}{\Gamma\left(1+\alpha_{i}\right)}\left(\zeta_{i}^{1} \eta_{i}+\wp_{1}\right)\right) \\
& +\mathcal{B}\left(\varrho_{i}\right)\left(\frac{1}{\Gamma\left(\varrho_{i}\right)} \frac{2 k^{\varrho_{i}}}{\Gamma(\varrho+1)} m\left(\left(\zeta_{i}^{2} \eta_{i}+\wp_{2}\right)\right)\right. \\
& \left.+\frac{1}{\Gamma\left(\alpha_{i}+\varrho_{i}\right)} \frac{2 k^{\varrho_{i}+\alpha_{i}}}{\Gamma\left(\varrho+\alpha_{i}+1\right)}\left(\left(\zeta_{i}^{1} \eta_{i}+\wp_{1}\right)\right)\right),
\end{aligned}
$$

and for $t \leq s$, we have

$$
\begin{aligned}
\left|\mathcal{T}_{i} u_{i}(t)\right|=\mid & -\frac{1-\varrho_{i}}{\mathcal{B}\left(\varrho_{i}\right)}\left(\sum_{1}^{m} \mathcal{F}_{i}\left(t, u_{i}(t)\right)+\mathcal{I}^{\alpha_{i}} \lambda_{i}\left(t, u_{i}(t)\right)\right) \\
& +\mathcal{B}\left(\varrho_{i}\right)\left(\frac{1}{\Gamma\left(\varrho_{i}\right)} \int_{0}^{1} \mathcal{G}_{i}(t, s) \sum_{1}^{m} \mathcal{F}_{i}\left(s, u_{i}(s)\right) d s\right. \\
& \left.+\frac{1}{\Gamma\left(\varrho_{i}+\alpha_{i}\right)} \int_{0}^{1} \mathcal{H}_{i}(t, s) \lambda_{i}\left(s, u_{i}(s)\right) d s\right) \mid \\
\leq & \frac{1-\varrho_{i}}{\mathcal{B}\left(\varrho_{i}\right)}\left(m\left(\zeta_{i}^{2} \eta_{i}+\wp_{2}\right)+\frac{1}{\Gamma\left(\alpha_{i}+1\right)}\left(\zeta_{i}^{1} \eta_{i}+\wp_{1}\right)\right) \\
& +\mathcal{B}\left(\varrho_{i}\right)\left(\frac{1}{\Gamma\left(\varrho_{i}\right)} \int_{0}^{1} \mathcal{G}_{i}(t, s) m\left(\left(\zeta_{i}^{2} \eta_{i}+\wp_{2}\right)\right) d s\right. \\
& \left.+\frac{1}{\Gamma\left(\varrho_{i}+\alpha_{i}\right)} \int_{0}^{1} \mathcal{H}_{i}(t, s)\left(\left(\zeta_{i}^{1} \eta_{i}+\wp_{1}\right)\right) d s\right) \\
\leq & \frac{1-\varrho_{i}}{\mathcal{B}\left(\varrho_{i}\right)}\left(m\left(\zeta_{i}^{2} \eta_{i}+\wp_{2}\right)+\frac{1}{\Gamma\left(\alpha_{i}+1\right)}\left(\zeta_{i}^{1} \eta_{i}+\wp_{1}\right)\right) \\
& +\mathcal{B}\left(\varrho_{i}\right)\left(\frac{1}{\Gamma\left(\varrho_{i}\right)} \frac{k^{\varrho_{i}}}{\Gamma(1+\varrho)} m\left(\left(\zeta_{i}^{2} \eta_{i}+\wp_{2}\right)\right)\right. \\
& \left.+\frac{1}{\Gamma\left(\varrho_{i}+\alpha_{i}\right)} \frac{k^{\varrho_{i}+\alpha_{i}}}{\Gamma\left(\varrho+\alpha_{i}+1\right)}\left(\left(\zeta_{i}^{1} \eta_{i}+\wp_{1}\right)\right)\right) .
\end{aligned}
$$

This implies $\mathcal{T}_{i} \mathcal{S}_{\eta_{i}} \subset \mathcal{S}_{\eta_{i}}$. Further, assuming that $u_{l}, u_{j} \in \mathcal{C}\left([0, k], \mathcal{R}_{e}\right)$ and $k \geq 1$, for $t \geq s \in$ $[0, k]$, we get

$$
\begin{aligned}
\left|\mathcal{T}_{i} u_{l}(t)-\mathcal{T}_{i} u_{j}(t)\right|=\mid & -\frac{1-\varrho_{i}}{\mathcal{B}\left(\varrho_{i}\right)}\left(\sum_{1}^{m} \mathcal{F}_{i}\left(t, u_{l}(t)\right)+\mathcal{I}^{\alpha_{i}} \lambda_{i}\left(t, u_{l}(t)\right)\right) \\
& +\mathcal{B}\left(\varrho_{i}\right)\left(\frac{1}{\Gamma\left(\varrho_{i}\right)} \int_{0}^{1} \mathcal{G}_{i}(t, s) \sum_{1}^{m} \mathcal{F}_{i}\left(s, u_{l}(s)\right) d s\right.
\end{aligned}
$$




$$
\begin{aligned}
+ & \left.\frac{1}{\Gamma\left(\varrho_{i}+\alpha_{i}\right)} \int_{0}^{1} \mathcal{H}_{i}(t, s) \lambda_{i}\left(s, u_{l}(s)\right) d s\right) \\
& -\left[\mathcal { B } ( \varrho _ { i } ) \left(\frac{1}{\Gamma\left(\varrho_{i}\right)} \int_{0}^{1} \mathcal{G}_{i}(t, s) \sum_{1}^{m} \mathcal{F}_{i}\left(s, u_{j}(s)\right) d s\right.\right. \\
& \left.+\frac{1}{\Gamma\left(\varrho_{i}+\alpha_{i}\right)} \int_{0}^{1} \mathcal{H}_{i}(t, s) \lambda_{i}\left(s, u_{j}(s)\right) d s\right) \\
& \left.-\frac{1-\varrho_{i}}{\mathcal{B}\left(\varrho_{i}\right)}\left(\sum_{1}^{m} \mathcal{F}_{i}\left(t, u_{j}(t)\right)+\mathcal{I}^{\alpha_{i}} \lambda_{i}\left(t, u_{j}(t)\right)\right)\right] \\
\leq & \frac{1-\varrho_{i}}{\mathcal{B}\left(\varrho_{i}\right)}\left(m\left(\zeta_{i}^{2}\left|u_{l}-u_{j}\right|\right)+\frac{1}{\Gamma\left(\alpha_{i}+1\right)}\left(\zeta_{i}^{1}\left|u_{l}-u_{j}\right|\right)\right) \\
& +\mathcal{B}\left(\varrho_{i}\right)\left(\frac{1}{\Gamma\left(\varrho_{i}\right)} \int_{0}^{1} \mathcal{G}_{i}(t, s) m\left(\left(\zeta_{i}^{2}\left|u_{l}-u_{j}\right|\right)\right) d s\right. \\
& \left.+\frac{1}{\Gamma\left(\varrho_{i}+\alpha_{i}\right)} \int_{0}^{1} \mathcal{H}_{i}(t, s)\left(\left(\zeta_{i}^{1}\left|u_{l}-u_{j}\right|\right)\right) d s\right) \\
\leq & {\left[\frac{1-\varrho_{i}}{\mathcal{B}\left(\varrho_{i}\right)}\left(m \zeta_{i}^{2}+\frac{1}{\Gamma\left(\alpha_{i}+1\right)} \zeta_{i}^{1}\right)+\mathcal{B}\left(\varrho_{i}\right)\left(\frac{1}{\Gamma\left(\varrho_{i}\right)} \frac{2 k^{\varrho_{i}}}{\Gamma(\varrho+1)} m \zeta_{i}^{2}\right.\right.} \\
& \left.\left.+\frac{1}{\Gamma\left(\varrho_{i}+\alpha_{i}\right)} \frac{2 k^{\varrho_{i}+\alpha_{i}}}{\Gamma\left(\varrho+\alpha_{i}+1\right)} \zeta_{i}^{1}\right)\right]\left|u_{l}-u_{j}\right|,
\end{aligned}
$$

and for $t \leq s \in[0, k]$, a calculation for $i=1,2, \ldots, m$ leads to

$$
\begin{aligned}
\left|\mathcal{T}_{i} u_{l}(t)-\mathcal{T}_{i} u_{j}(t)\right|=\mid & -\frac{1-\varrho_{i}}{\mathcal{B}\left(\varrho_{i}\right)}\left(\sum_{1}^{m} \mathcal{F}_{i}\left(t, u_{l}(t)\right)+\mathcal{I}^{\alpha_{i}} \lambda_{i}\left(t, u_{l}(t)\right)\right) \\
& +\mathcal{B}\left(\varrho_{i}\right)\left(\frac{1}{\Gamma\left(\varrho_{i}\right)} \int_{0}^{1} \mathcal{G}_{i}(t, s) \sum_{1}^{m} \mathcal{F}_{i}\left(s, u_{l}(s)\right) d s\right. \\
& \left.+\frac{1}{\Gamma\left(\varrho_{i}+\alpha_{i}\right)} \int_{0}^{1} \mathcal{H}_{i}(t, s) \lambda_{i}\left(s, u_{l}(s)\right) d s\right) \\
& -\left[-\frac{1-\varrho_{i}}{\mathcal{B}\left(\varrho_{i}\right)}\left(\sum_{1}^{m} \mathcal{F}_{i}\left(t, u_{j}(t)\right)+\mathcal{I}^{\alpha_{i}} \lambda_{i}\left(t, u_{j}(t)\right)\right)\right. \\
& +\mathcal{B}\left(\varrho_{i}\right)\left(\frac{1}{\Gamma\left(\varrho_{i}\right)} \int_{0}^{1} \mathcal{G}_{i}(t, s) \sum_{1}^{m} \mathcal{F}_{i}\left(s, u_{j}(s)\right) d s\right. \\
& \left.\left.+\frac{1}{\Gamma\left(\varrho_{i}+\alpha_{i}\right)} \int_{0}^{1} \mathcal{H}_{i}(t, s) \lambda_{i}\left(s, u_{j}(s)\right) d s\right)\right] \mid \\
\leq & \frac{1-\varrho_{i}}{\mathcal{B}\left(\varrho_{i}\right)}\left(m\left(\zeta_{i}^{2}\left|u_{l}-u_{j}\right|\right)+\frac{1}{\Gamma\left(\alpha_{i}+1\right)}\left(\zeta_{i}^{1}\left|u_{l}-u_{j}\right|\right)\right) \\
& +\mathcal{B}\left(\varrho_{i}\right)\left(\frac{1}{\Gamma\left(\varrho_{i}\right)} \int_{0}^{1} \mathcal{G}_{i}(t, s) m\left(\left(\zeta_{i}^{2}\left|u_{l}-u_{j}\right|\right)\right) d s\right. \\
& \left.+\frac{1}{\Gamma\left(\varrho_{i}+\alpha_{i}\right)} \int_{0}^{1} \mathcal{H}_{i}(t, s)\left(\left(\zeta_{i}^{1}\left|u_{l}-u_{j}\right|\right)\right) d s\right)
\end{aligned}
$$




$$
\begin{aligned}
\leq & {\left[\frac{1-\varrho_{i}}{\mathcal{B}\left(\varrho_{i}\right)}\left(m \zeta_{i}^{2}+\frac{1}{\Gamma\left(\alpha_{i}+1\right)} \zeta_{i}^{1}\right)+\mathcal{B}\left(\varrho_{i}\right)\left(\frac{1}{\Gamma\left(\varrho_{i}\right)} \frac{k^{\varrho_{i}}}{\Gamma(\varrho+1)} m \zeta_{i}^{2}\right.\right.} \\
& \left.\left.+\frac{1}{\Gamma\left(\varrho_{i}+\alpha_{i}\right)} \frac{k^{\varrho_{i}+\alpha_{i}}}{\Gamma\left(\varrho+\alpha_{i}+1\right)} \zeta_{i}^{1}\right)\right]\left|u_{l}-u_{j}\right| \\
\leq & \eta_{i}\left|u_{l}-u_{j}\right| .
\end{aligned}
$$

If $\eta_{i}<1$, where $\eta_{i}$ are given by (18), then $\mathcal{T}_{i}$ are contraction operators, and by Banach's fixed point theorem the sequential hybrid fractional-order system (1) has a unique solution, represented by the fixed points of $\mathcal{T}_{i}$.

Theorem 2.3 Under the assumptions of Lemma 2.2, the sequential hybrid system of FDEs (1) has a solution.

Proof In Lemma 2.2, we proved that $\mathcal{T}_{i}$ are bounded. Furthermore, let $t_{1}, t_{2} \in[0, k]$ with $t_{2}>t_{1}$ and $k \leq 1$. In the case $t \geq s$, we have

$$
\begin{aligned}
& \left|\mathcal{T}_{i} u\left(t_{2}\right)-\mathcal{T}_{i} u\left(t_{1}\right)\right| \\
& =\mid-\frac{1-\varrho_{i}}{\mathcal{B}\left(\varrho_{i}\right)}\left(\sum_{1}^{m} \mathcal{F}_{i}\left(t_{2}, u\left(t_{2}\right)\right)+\mathcal{I}^{\alpha_{i}} \lambda_{i}\left(t_{2}, u\left(t_{2}\right)\right)\right) \\
& +\mathcal{B}\left(\varrho_{i}\right)\left(\frac{1}{\Gamma\left(\varrho_{i}\right)} \int_{0}^{1} \mathcal{G}_{i}\left(t_{2}, s\right) \sum_{1}^{m} \mathcal{F}_{i}(s, u(s)) d s\right. \\
& \left.+\frac{1}{\Gamma\left(\varrho_{i}+\alpha_{i}\right)} \int_{0}^{1} \mathcal{H}_{i}\left(t_{2}, s\right) \lambda_{i}(s, u(s)) d s\right) \\
& -\left[-\frac{1-\varrho_{i}}{\mathcal{B}\left(\varrho_{i}\right)}\left(\sum_{1}^{m} \mathcal{F}_{i}\left(t_{1}, u\left(t_{1}\right)\right)+\mathcal{I}^{\alpha_{i}} \lambda_{i}\left(t_{1}, u\left(t_{1}\right)\right)\right)\right. \\
& +\mathcal{B}\left(\varrho_{i}\right)\left(\frac{1}{\Gamma\left(\varrho_{i}\right)} \int_{0}^{1} \mathcal{G}_{i}\left(t_{1}, s\right) \sum_{1}^{m} \mathcal{F}_{i}(s, u(s)) d s\right. \\
& \left.\left.+\frac{1}{\Gamma\left(\varrho_{i}+\alpha_{i}\right)} \int_{0}^{1} \mathcal{H}_{i}\left(t_{1}, s\right) \lambda_{i}(s, u(s)) d s\right)\right] \mid \\
& \leq \frac{1-\varrho_{i}}{\mathcal{B}\left(\varrho_{i}\right)}\left(\sum_{1}^{m}\left|\mathcal{F}_{i}\left(t_{2}, u\left(t_{2}\right)\right)-\mathcal{F}_{i}\left(t_{1}, u\left(t_{1}\right)\right)\right|+\mathcal{I}^{\alpha_{i}}\left|\lambda_{i}\left(t_{2}, u\left(t_{2}\right)\right)-\lambda_{i}\left(t_{1}, u\left(t_{1}\right)\right)\right|\right) \\
& +\mathcal{B}\left(\varrho_{i}\right)\left(\frac{1}{\Gamma\left(\varrho_{i}\right)}\left|\int_{0}^{t_{2}}\left(t_{2}-s\right)^{\varrho_{i}-1}-\int_{0}^{t_{1}}\left(t_{1}-s\right)^{\varrho_{i}-1}\right| \sum_{1}^{m}\left|\mathcal{F}_{i}(s, u(s))\right| d s\right. \\
& \left.+\frac{1}{\Gamma\left(\varrho_{i}+\alpha_{i}\right)}\left|\int_{0}^{t_{2}}\left(t_{2}-s\right)^{\varrho_{i}+\alpha-1}-\int_{0}^{t_{1}}\left(t_{1}-s\right)^{\varrho_{i}+\alpha-1}\right|\left|\lambda_{i}(s, u(s))\right| d s\right) \\
& \leq \frac{1-\varrho_{i}}{\mathcal{B}\left(\varrho_{i}\right)}\left(\sum_{1}^{m}\left|\mathcal{F}_{i}\left(t_{2}, u\left(t_{2}\right)\right)-\mathcal{F}_{i}\left(t_{1}, u\left(t_{1}\right)\right)\right|+\mathcal{I}^{\alpha_{i}}\left|\lambda_{i}\left(t_{2}, u\left(t_{2}\right)\right)-\lambda_{i}\left(t_{1}, u\left(t_{1}\right)\right)\right|\right) \\
& +\mathcal{B}\left(\varrho_{i}\right)\left(\frac{1}{\Gamma\left(\varrho_{i}+1\right)}\left|t_{2}^{\varrho_{i}}-t_{1}^{\varrho_{i}}\left(\zeta_{i}^{2} \eta_{i}+\wp_{2}\right)+\frac{1}{\Gamma\left(\varrho_{i}+\alpha_{i}+1\right)}\right| t_{2}^{\varrho_{i}+\alpha}\right. \\
& \left.-t_{1}^{\varrho_{i}+\alpha} \mid\left(\zeta_{i}^{1} \eta_{i}+\wp_{1}\right)\right) \text {. }
\end{aligned}
$$


This implies that $\mathcal{T}_{i} u\left(t_{2}\right) \rightarrow \mathcal{T}_{i} u\left(t_{1}\right)$ as $t_{2} \rightarrow t_{1}$. This implies $\left|\mathcal{T}_{i} u_{i}\left(t_{2}\right)-\mathcal{T}_{i} u_{i}\left(t_{1}\right)\right| \rightarrow 0$ as $t_{2} \rightarrow t_{1}$. Hence $\mathcal{T}_{i}$ are equicontinuous operators for $t \geq s$. The case $t \leq s$ is similar, and thus we omit it. Next, for any $u \in\left\{u \in \mathcal{C}\left([0, k], \mathcal{R}_{e}\right): u=\hbar \mathcal{T}_{i}(u)\right.$, for $\left.\hbar \in[0,1]\right\}$, we have

$$
\begin{aligned}
\|u\|= & \left|\mathcal{T}_{i} u_{i}(t)\right| \\
= & \mid-\frac{1-\varrho_{i}}{\mathcal{B}\left(\varrho_{i}\right)}\left(\sum_{1}^{m} \mathcal{F}_{i}\left(t, u_{i}(t)\right)+\mathcal{I}^{\alpha_{i}} \lambda_{i}\left(t, u_{i}(t)\right)\right) \\
& +\mathcal{B}\left(\varrho_{i}\right)\left(\frac{1}{\Gamma\left(\varrho_{i}\right)} \int_{0}^{1} \mathcal{G}_{i}(t, s) \sum_{1}^{m} \mathcal{F}_{i}\left(s, u_{i}(s)\right) d s\right. \\
& \left.+\frac{1}{\Gamma\left(\varrho_{i}+\alpha_{i}\right)} \int_{0}^{1} \mathcal{H}_{i}(t, s) \lambda_{i}\left(s, u_{i}(s)\right) d s\right) \mid \\
\leq & \frac{1-\varrho_{i}}{\mathcal{B}\left(\varrho_{i}\right)}\left(m\left(\zeta_{i}^{2} \eta_{i}+\wp_{2}\right)+\frac{1}{\Gamma\left(\alpha_{i}+1\right)}\left(\zeta_{i}^{1} \eta_{i}+\wp_{1}\right)\right) \\
& +\mathcal{B}\left(\varrho_{i}\right)\left(\frac{1}{\Gamma\left(\varrho_{i}\right)} \int_{0}^{1} \mathcal{G}_{i}(t, s) m\left(\left(\zeta_{i}^{2} \eta_{i}+\wp_{2}\right)\right) d s\right. \\
& \left.+\frac{1}{\Gamma\left(\varrho_{i}+\alpha_{i}\right)} \int_{0}^{1} \mathcal{H}_{i}(t, s)\left(\left(\zeta_{i}^{1} \eta_{i}+\wp_{1}\right)\right) d s\right) \\
\leq & \frac{1-\varrho_{i}}{\mathcal{B}\left(\varrho_{i}\right)}\left(m\left(\zeta_{i}^{2} \eta_{i}+\wp_{2}\right)+\frac{1}{\Gamma\left(\alpha_{i}+1\right)}\left(\zeta_{i}^{1} \eta_{i}+\wp_{1}\right)\right) \\
& +\mathcal{B}\left(\varrho_{i}\right)\left(\frac{1}{\Gamma\left(\varrho_{i}\right)} \frac{k^{\varrho_{i}}}{\Gamma(\varrho+1)} m\left(\left(\zeta_{i}^{2} \eta_{i}+\wp_{2}\right)\right)\right. \\
& \left.+\frac{1}{\Gamma\left(\varrho_{i}+\alpha_{i}\right)} \frac{k^{\alpha_{i}+\varrho_{i}}}{\Gamma\left(\varrho+\alpha_{i}+1\right)}\left(\left(\zeta_{i}^{1} \eta_{i}+\wp_{1}\right)\right)\right) \\
= & \Lambda_{1}+\Lambda_{2}\|u\|,
\end{aligned}
$$

where

$$
\begin{aligned}
\Lambda_{i}^{1}= & \frac{1-\varrho_{i}}{\mathcal{B}\left(\varrho_{i}\right)}\left(m \zeta_{i}^{2}+\frac{1}{\Gamma\left(\alpha_{i}+1\right)} \zeta_{i}^{1}\right)+\mathcal{B}\left(\varrho_{i}\right)\left(\frac{1}{\Gamma\left(\varrho_{i}\right)} \frac{k^{\varrho_{i}}}{\Gamma(\varrho+1)} m \zeta_{i}^{2}\right. \\
& \left.+\frac{1}{\Gamma\left(\varrho_{i}+\alpha_{i}\right)} \frac{k^{\varrho_{i}+\alpha_{i}}}{\Gamma\left(\varrho+\alpha_{i}+1\right)} \zeta_{i}^{1}\right)
\end{aligned}
$$

and

$$
\begin{aligned}
\Lambda_{i}^{2}= & \frac{1-\varrho_{i}}{\mathcal{B}\left(\varrho_{i}\right)}\left(m \wp_{2}+\frac{1}{\Gamma\left(\alpha_{i}+1\right)} \wp_{1}\right)+\mathcal{B}\left(\varrho_{i}\right)\left(\frac{1}{\Gamma\left(\varrho_{i}\right)} \frac{k^{\varrho_{i}}}{\Gamma(\varrho+1)} m \wp_{2}\right. \\
& \left.+\frac{1}{\Gamma\left(\varrho_{i}+\alpha_{i}\right)} \frac{k^{\varrho_{i}+\alpha_{i}}}{\Gamma\left(\varrho+\alpha_{i}+1\right)} \wp_{1}\right)
\end{aligned}
$$

for $i=1,2, \ldots, m$. With the help of (31), (32), and (33) we have

$$
\|u\| \leq \frac{\Lambda_{i}^{1}}{1-\Lambda_{i}^{2}}
$$


for $i=1,2, \ldots, m$. Hence the requirement of the Leray-Schauder alternative theorem is ensured, and therefore the system of sequential hybrid FDEs (1) has a solution.

\section{H-U-stability}

Here we consider the H-U stability of system (15). The following definition plays a vital role in the stability.

Definition 3.1 The fractional-order integral system (15) is $\mathrm{H}$-U-stable if for some $\zeta_{i}>0$, there exist $\Delta_{i}>0$, for each solution $u_{i}$ with

$$
\left\|u_{i}-\mathcal{T}_{i} u_{i}\right\|_{1}<\Delta_{i}
$$

there are $\bar{u}_{i}(t)$ of the operators system (15) with

$$
\bar{u}_{i}(t)=\mathcal{T}_{i} \bar{u}_{i}(t)
$$

such that

$$
\left\|u_{i}-\bar{u}_{i}\right\|<\Delta_{i} \zeta_{i}
$$

for all $i=1,2, \ldots, m$.

Theorem 3.2 With assumptions of Lemma 2.2, the integral system (15) is H-U stable, that is, the sequential hybrid system of FDEs (1) is $\mathrm{H}$-U stable.

Proof Let $u_{i} \in \mathcal{C}$ satisfy inequality (35), and let $\bar{u}_{i} \in \mathcal{C}$ of system (1) satisfy (15). Also,

$$
\begin{aligned}
\left|\mathcal{T}_{i} u_{i}(t)-\mathcal{T}_{i} \bar{u}^{*}(t)\right|=\mid & -\frac{1-\varrho_{i}}{\mathcal{B}\left(\varrho_{i}\right)}\left(\sum_{1}^{m} \mathcal{F}_{i}\left(t, u_{i}(t)\right)+\mathcal{I}^{\alpha_{i}} \lambda_{i}\left(t, u_{i}(t)\right)\right) \\
& +\mathcal{B}\left(\varrho_{i}\right)\left(\frac{1}{\Gamma\left(\varrho_{i}\right)} \int_{0}^{1} \mathcal{G}_{i}(t, s) \sum_{1}^{m} \mathcal{F}_{i}\left(s, u_{i}(s)\right) d s\right. \\
& \left.+\frac{1}{\Gamma\left(\varrho_{i}+\alpha_{i}\right)} \int_{0}^{1} \mathcal{H}_{i}(t, s) \lambda_{i}\left(s, u_{i}(s)\right) d s\right) \\
& -\left[-\frac{1-\varrho_{i}}{\mathcal{B}\left(\varrho_{i}\right)}\left(\sum_{1}^{m} \mathcal{F}_{i}\left(t, \bar{u}^{*}(t)\right)+\mathcal{I}^{\alpha_{i}} \lambda_{i}\left(t, \bar{u}^{*}(t)\right)\right)\right. \\
& +\mathcal{B}\left(\varrho_{i}\right)\left(\frac{1}{\Gamma\left(\varrho_{i}\right)} \int_{0}^{1} \mathcal{G}_{i}(t, s) \sum_{1}^{m} \mathcal{F}_{i}\left(s, \bar{u}^{*}(s)\right) d s\right. \\
& \left.\left.+\frac{1}{\Gamma\left(\varrho_{i}+\alpha_{i}\right)} \int_{0}^{1} \mathcal{H}_{i}(t, s) \lambda_{i}\left(s, \bar{u}^{*}(s)\right) d s\right)\right] \mid \\
\leq & \frac{1-\varrho_{i}}{\mathcal{B}\left(\varrho_{i}\right)}\left(m\left(\zeta_{i}^{2}\left|u_{i}-\bar{u}^{*}\right|\right)+\frac{1}{\Gamma\left(\alpha_{i}+1\right)}\left(\zeta_{i}^{1}\left|u_{i}-\bar{u}^{*}\right|\right)\right) \\
& +\mathcal{B}\left(\varrho_{i}\right)\left(\frac{1}{\Gamma\left(\varrho_{i}\right)} \int_{0}^{1} \mathcal{G}_{i}(t, s) m\left(\left(\zeta_{i}^{2}\left|u_{i}-\bar{u}^{*}\right|\right)\right) d s\right.
\end{aligned}
$$




$$
\begin{aligned}
& \left.+\frac{1}{\Gamma\left(\varrho_{i}+\alpha_{i}\right)} \int_{0}^{1} \mathcal{H}_{i}(t, s)\left(\left(\zeta_{i}^{1}\left|u_{i}-\bar{u}^{*}\right|\right)\right) d s\right) \\
\leq & {\left[\frac{1-\varrho_{i}}{\mathcal{B}\left(\varrho_{i}\right)}\left(m \zeta_{i}^{2}+\frac{1}{\Gamma\left(\alpha_{i}+1\right)} \zeta_{i}^{1}\right)+\mathcal{B}\left(\varrho_{i}\right)\left(\frac{1}{\Gamma\left(\varrho_{i}\right)} \frac{2 k^{\varrho_{i}}}{\Gamma(\varrho+1)} m \zeta_{i}^{2}\right.\right.} \\
& \left.\left.+\frac{1}{\Gamma\left(\varrho_{i}+\alpha_{i}\right)} \frac{2 k^{\varrho_{i}+\alpha_{i}}}{\Gamma\left(\varrho+\alpha_{i}+1\right)} \zeta_{i}^{1}\right)\right]\left|u_{i}-\bar{u}^{*}\right| .
\end{aligned}
$$

For $t \leq s \in[0, k]$, a calculation leads to

$$
\begin{aligned}
\left|\mathcal{T}_{i} u_{i}(t)-\mathcal{T}_{i} \bar{u}^{*}(t)\right| & \mid \frac{1-\varrho_{i}}{\mathcal{B}\left(\varrho_{i}\right)}\left(\sum_{1}^{m} \mathcal{F}_{i}\left(t, u_{i}(t)\right)+\mathcal{I}^{\alpha_{i}} \lambda_{i}\left(t, u_{i}(t)\right)\right) \\
& +\mathcal{B}\left(\varrho_{i}\right)\left(\frac{1}{\Gamma\left(\varrho_{i}\right)} \int_{0}^{1} \mathcal{G}_{i}(t, s) \sum_{1}^{m} \mathcal{F}_{i}\left(s, u_{i}(s)\right) d s\right. \\
& \left.+\frac{1}{\Gamma\left(\varrho_{i}+\alpha_{i}\right)} \int_{0}^{1} \mathcal{H}_{i}(t, s) \lambda_{i}\left(s, u_{i}(s)\right) d s\right) \\
& -\left[-\frac{1-\varrho_{i}}{\mathcal{B}\left(\varrho_{i}\right)}\left(\sum_{1}^{m} \mathcal{F}_{i}\left(t, \bar{u}^{*}(t)\right)+\mathcal{I}^{\alpha_{i}} \lambda_{i}\left(t, \bar{u}^{*}(t)\right)\right)\right. \\
& +\mathcal{B}\left(\varrho_{i}\right)\left(\frac{1}{\Gamma\left(\varrho_{i}\right)} \int_{0}^{1} \mathcal{G}_{i}(t, s) \sum_{1}^{m} \mathcal{F}_{i}\left(s, \bar{u}^{*}(s)\right) d s\right. \\
& \left.\left.+\frac{1}{\Gamma\left(\alpha_{i}+\varrho_{i}\right)} \int_{0}^{1} \mathcal{H}_{i}(t, s) \lambda_{i}\left(s, \bar{u}^{*}(s)\right) d s\right)\right] \mid \\
\leq & \frac{1-\varrho_{i}}{\mathcal{B}\left(\varrho_{i}\right)}\left(m\left(\zeta_{i}^{2}\left|u_{i}-\bar{u}^{*}\right|\right)+\frac{1}{\Gamma\left(\alpha_{i}+1\right)}\left(\zeta_{i}^{1}\left|u_{i}-\bar{u}^{*}\right|\right)\right) \\
& +\mathcal{B}\left(\varrho_{i}\right)\left(\frac{1}{\Gamma\left(\varrho_{i}\right)} \int_{0}^{1} \mathcal{G}_{i}(t, s) m\left(\left(\zeta_{i}^{2}\left|u_{i}-\bar{u}^{*}\right|\right)\right) d s\right. \\
& \left.+\frac{1}{\Gamma\left(\varrho_{i}+\alpha_{i}\right)} \int_{0}^{1} \mathcal{H}_{i}(t, s)\left(\left(\zeta_{i}^{1}\left|u_{i}-\bar{u}^{*}\right|\right)\right) d s\right) \\
\leq & \frac{1-\varrho_{i}}{\mathcal{B}\left(\varrho_{i}\right)}\left(m \zeta_{i}^{2}+\frac{1}{\Gamma\left(\varrho_{i}+1\right)} \zeta_{i}^{1}\right)+\mathcal{B}\left(\varrho_{i}\right)\left(\frac{1}{\Gamma\left(\varrho_{i}\right)} \frac{k^{\varrho_{i}+\alpha_{i}}}{\Gamma(\varrho+1)} m \zeta_{i}^{2}\right. \\
& \left.\left.+\varrho+\alpha_{i}+1\right)\right]\left|u_{i}-\bar{u}^{*}\right| \\
& \left.+\frac{1}{\Gamma}\right)
\end{aligned}
$$

Let $\eta_{i}<1$, where $\eta_{i}$ are defined in (18) for $i=1,2, \ldots, m$. With the help of (35), (36), (38), and (40) consider the norm

$$
\begin{aligned}
\left\|u_{i}-\bar{u}_{i}^{*}\right\| & =\left\|u_{i}-\mathcal{T}_{i} u_{i}+\mathcal{T}_{i} u_{i}-\bar{u}_{i}^{*}\right\| \\
& \leq\left\|u_{i}-\mathcal{T}_{i} u_{i}\right\|+\left\|\mathcal{T}_{i} u_{i}-\mathcal{T}_{i} \bar{u}_{i}^{*}\right\| \\
& \leq \Delta_{i}+\eta_{i}\left\|u_{i}-\bar{u}_{i}^{*}\right\|
\end{aligned}
$$


for $i=1,2, \ldots, m$. This further implies that

$$
\left\|u_{i}-\bar{u}_{i}^{*}\right\| \leq \frac{\Delta_{i}}{1-\eta_{i}}
$$

with $\zeta_{i}=\frac{1}{1-\eta_{i}}$. Therefore system (15) is H-U stable. This ultimately ensures the stability of the sequential hybrid system of FDEs (1).

\section{Application}

Here we present an application of problem (1) and provide its numerical simulations. The following system of four equations is a generalization of the smoking model with relapse effect from quit smokers to the potential smoker [59]. Here $\mathcal{P}$ is a potential smoker, $\mathcal{L}$ is a slight smoker, $\mathcal{S}$ is a smoker, and $\mathcal{Q}$ is quit smokers. We have

$$
\begin{aligned}
& { }^{A B C} \mathcal{D}_{0}^{\varrho_{1}} \mathcal{P}=\Lambda^{*}+\varrho \mathcal{Q}-2 \frac{\beta^{*} \mathcal{P} \mathcal{L}}{\mathcal{P}+\mathcal{L}}-(\mu+d) \mathcal{P}, \\
& { }^{A B C} \mathcal{D}_{0}^{\varrho_{2}} \mathcal{L}=2 \frac{\beta^{*} \mathcal{P} \mathcal{L}}{\mathcal{P}+\mathcal{L}}-(\xi+\mu+d) \mathcal{L}, \\
& { }^{A B C} \mathcal{D}_{0}^{\varrho_{3}} \mathcal{S}=\xi \mathcal{L}-(\xi+\mu+\delta) \mathcal{S} \\
& { }^{A B C} \mathcal{D}_{0}^{\varrho_{4}} \mathcal{Q}=\delta \mathcal{S}-(\xi+\mu+\gamma) \mathcal{Q} .
\end{aligned}
$$

Here $\varrho_{i} \in(0,1], i=1,2, \ldots, 6,\left(u_{1}, u_{2}, u_{3}, u_{4}\right)=\left(\mathcal{P}, \mathcal{L}_{1}, \mathcal{S}_{2}, \mathcal{Q}\right), \mathcal{G}_{1}(t, \mathcal{P})=\Lambda^{*}+\varrho \mathcal{Q}-2 \frac{\beta^{*} \mathcal{P} \mathcal{L}}{\mathcal{P}+\mathcal{L}}-$ $(\mu+d) \mathcal{P}, \mathcal{G}_{2}(t, \mathcal{L})=2 \frac{\beta^{*} \mathcal{P} \mathcal{L}}{\mathcal{P}+\mathcal{L}}-(\xi+\mu+d) \mathcal{L}, \mathcal{G}_{3}(t, \mathcal{S})=\xi \mathcal{L}-(\xi+\mu+\delta) \mathcal{S}$, and $\mathcal{G}_{4}(t, \mathcal{Q})=$ $\delta \mathcal{S}-(\xi+\mu+\gamma) \mathcal{Q}$.

\subsection{Numerical scheme}

Let us consider

$$
{ }^{A B C}{ }_{0} D_{t}^{\varrho_{1}, \varrho_{2}} \mathcal{P}(t)=\mathcal{H}(t, \mathcal{P}(t)),
$$

where $\mathcal{P}(0)=\mathcal{P}_{0}$. Applying the $\mathrm{AB}$ fractional integral, we get

$$
\mathcal{P}(t)=\mathcal{P}(0)+\frac{1-\varrho_{1}}{A B\left(\varrho_{1}\right)} \mathcal{H}(t, \mathcal{P}(t))+\frac{\varrho_{1}}{A B\left(\varrho_{1}\right) \Gamma \varrho_{1}} \int_{0}^{t} \zeta^{\varrho_{2}-1}(t-\zeta)^{\varrho_{1}-1} \mathcal{H}(\zeta, \mathcal{P}(\zeta)) d \zeta
$$

Replacing $(t)$ by $t_{n+1}$, we have

$$
\begin{aligned}
\mathcal{P}_{n+1}= & \mathcal{P}(0)+\frac{1-\varrho_{1}}{A B\left(\varrho_{1}\right)} \mathcal{H}\left(t_{n}, \mathcal{P}\left(t_{n}\right)\right) \\
& +\frac{\varrho_{1}}{A B\left(\varrho_{1}\right) \Gamma \varrho_{1}} \int_{0}^{t_{n+1}} \zeta^{\varrho_{2}-1}\left(t_{n+1}-\zeta\right)^{\varrho_{1}-1} \mathcal{H}(\zeta, \mathcal{P}(\zeta)) d \zeta
\end{aligned}
$$

and

$$
\begin{aligned}
H(t, \mathcal{P}(t)) & =\frac{H\left(t_{k}, \mathcal{P}\left(t_{k}\right)\left(y-t_{k-1}\right)\right.}{t_{k}-t_{k-1}}-\frac{H\left(t_{k-1}, \mathcal{P}\left(t_{k-1}\right)\right)\left(y-t_{k}\right)}{t_{k}-t_{k-1}} \\
& =\frac{H\left(t_{k}, \mathcal{P}_{k}\right)\left(y-t_{k-1}\right)}{h}-\frac{H\left(t_{k-1}, \mathcal{P}_{k-1}\right)\left(y-t_{k}\right)}{h} .
\end{aligned}
$$


By applying the Lagrange polynomial we further have

$$
\begin{aligned}
\mathcal{P}_{n+1}= & \mathcal{P}(0)+\frac{1-\varrho_{1}}{A B\left(\varrho_{1}\right)} \mathcal{H}\left(t_{n}, \mathcal{P}\left(t_{n}\right)\right) \\
& +\frac{\varrho_{1}}{A B\left(\varrho_{1}\right) \Gamma \varrho_{1}} \sum_{i=1}^{n}\left[\frac{\mathcal{H}\left(t_{i}, \mathcal{P}\left(t_{i}\right)\right)}{h} \int_{t_{k}}^{t_{k+1}}\left(\zeta-t_{i-1}\right)\left(t_{n+1}-\zeta\right)^{\varrho_{1}-1} d \zeta\right. \\
& \left.-\frac{\mathcal{H}\left(t_{i-1}, \mathcal{P}\left(t_{i-1}\right)\right)}{h} \int_{t_{k}}^{t_{n+1}}\left(\zeta-t_{i}\right)\left(t_{n+1}-\zeta\right)^{\varrho_{1}-1} d \zeta\right]
\end{aligned}
$$

Now solving the integrals, we get

$$
\begin{aligned}
\mathcal{P}_{n+1}= & \mathcal{P}(0)+\frac{1-\varrho_{1}}{A B\left(\varrho_{1}\right)} \mathcal{H}\left(t_{n}, \mathcal{P}\left(t_{n}\right)\right) \\
& +\frac{\varrho_{1} h^{\varrho_{1}}}{\Gamma\left(\varrho_{1}+2\right)} \sum_{i=1}^{n}\left[\mathcal { H } ( t _ { i } , \mathcal { P } ( t _ { i } ) ) \left((n-i+1)^{\varrho_{1}}\left(n+2-i+\varrho_{1}\right)\right.\right. \\
& \left.-(n-i)^{\varrho_{1}}\left(n+2-i+2 \varrho_{1}\right)\right) \\
& \left.-\mathcal{H}\left(t_{i-1}, \mathcal{P}_{i-1}\right)\left((n-i+1)^{\varrho_{1}+1}-\left(n-i+1+\varrho_{1}\right)(n-i)^{\varrho_{1}}\right)\right] .
\end{aligned}
$$

Replacing the value of $\mathcal{H}(t, \mathcal{P}(t))$ by the functions, we obtain the following numerical scheme:

$$
\begin{aligned}
& \mathbb{P}_{n+1}= \mathbb{P}(0)+\varrho_{2} t^{\varrho_{2}-1} \frac{1-\varrho_{1}}{A B\left(\varrho_{1}\right)} \mathcal{G}_{1}\left(t_{n}, \mathbb{P}\left(t_{n}\right)\right)+\varrho_{2} t^{\varrho_{2}-1} \frac{\varrho_{2} h^{\varrho_{1}}}{\Gamma\left(\varrho_{1}+2\right)} \\
& \times \sum_{i=1}^{n}\left[\mathcal{G}_{1}\left(t_{i}, \mathbb{P}\left(t_{i}\right)\right)\left((n-i+1)^{\varrho_{1}}\left(\varrho_{1}+n+2-i\right)-(n-i)^{\varrho_{1}}\left(-i+2 \varrho_{1}+n+2\right)\right)\right. \\
&\left.-\mathcal{G}_{1}\left(t_{i-1}, \mathbb{P}_{i-1}\right)\left((n+1-i)^{\varrho_{1}+1}-\left(n+\varrho_{1}-i+1\right)(n-i)^{\varrho_{1}}\right)\right] \\
& \mathbb{L}_{n+1}= \mathbb{L}(0)+\varrho_{2} t^{\varrho_{2}-1} \frac{1-\varrho_{1}}{A B\left(\varrho_{1}\right)} \mathcal{G}_{2}\left(t_{n}, \mathbb{L}_{n}\right)+\varrho_{2} t^{\varrho_{2}-1} \frac{\varrho_{2} h^{\varrho_{1}}}{\Gamma\left(\varrho_{1}+2\right)} \\
& \times \sum_{i=1}^{n}\left[\mathcal{G}_{2}\left(t_{i}, \mathbb{L}_{i}\right)\left((n-i+1)^{\varrho_{1}}\left(n+2-i+\varrho_{1}\right)-(n-i)^{\varrho_{1}}\left(n+2 \varrho_{1}+2-i\right)\right)\right. \\
&\left.-\mathcal{G}_{2}\left(t_{i-1}, \mathbb{L}_{i-1}\right)\left((-i+n+1)^{\varrho_{1}+1}-\left(n-i+\varrho_{1}+1\right)(n-i)^{\varrho_{1}}\right)\right] \\
& \mathbb{S}_{n+1}= \mathbb{S}(0)+\varrho_{2} t^{\varrho_{2}-1} \frac{1-\varrho_{1}}{A B\left(\varrho_{1}\right)} \mathcal{G}_{3}\left(t_{n}, \mathbb{S}_{n}\right)+\varrho_{2} t^{\varrho_{2}-1} \frac{\varrho_{2} h^{\varrho_{1}}}{\Gamma\left(\varrho_{1}+2\right)} \\
& \times \sum_{i=1}^{n}\left[\mathcal{G}_{3}\left(t_{i}, \mathbb{S}_{i}\right)\left((n-i+1)^{\varrho_{1}}\left(n+\varrho_{1}+2-i\right)-(n-i)^{\varrho_{1}}\left(n+2 \varrho_{1}+2-i\right)\right)\right. \\
&\left.-\mathcal{G}_{3}\left(t_{i-1}, \mathbb{S}_{i-1}\right)\left((n-i+1)^{1+\varrho_{1}}-\left(n-i+1+\varrho_{1}\right)(n-i)^{\varrho_{1}}\right)\right] \\
&\left.-\mathcal{G}_{4}\left(t_{i-1}, \mathbb{Q}_{i-1}\right)\left((n+1-i)^{\varrho_{1}+1}-\left(n+\varrho_{1}-i+1\right)(n-i)^{\varrho_{1}}\right)\right] \\
& \times \sum_{i=1}^{n}\left[\mathcal{G}_{4}\left(t_{i}, \mathbb{Q}_{i}\right)\left((n-i+1)^{\varrho_{1}}\left(n+2-i+\varrho_{1}\right)-(n-i)^{\varrho_{1}}\left(n+2-i+2 \varrho_{1}\right)\right)\right. \\
& \mathbb{Q}_{n+1} t^{\varrho_{2}-1} \frac{1-\varrho_{1}}{A B\left(\varrho_{1}\right)} \mathcal{G}_{4}\left(t_{n}, \mathbb{Q}_{n}\right)+\varrho_{2} t^{\varrho_{2}-1} \frac{\varrho_{2} h^{\varrho_{1}}}{\Gamma\left(\varrho_{1}+2\right)} \\
& \mathbb{Q}(0)
\end{aligned}
$$




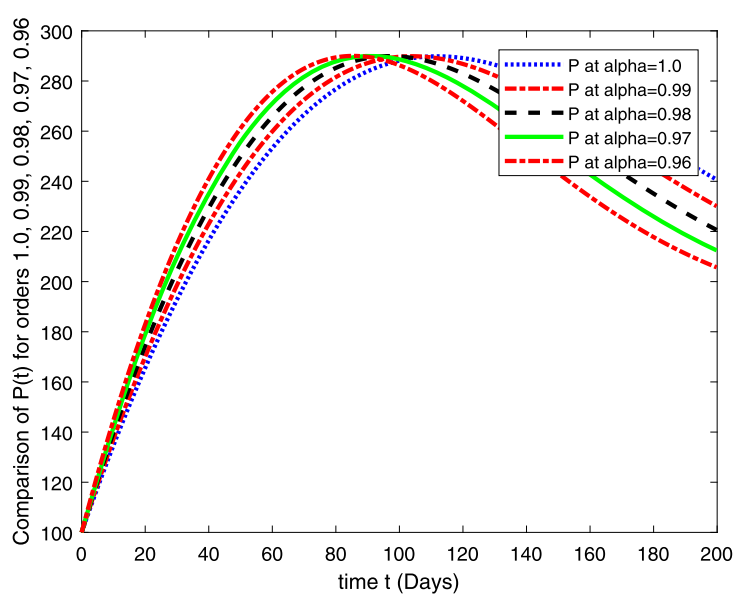

Figure 1 Potential smokers $\mathcal{P}(t)$ for orders 1.0, 0.99, 0.98, 0.97

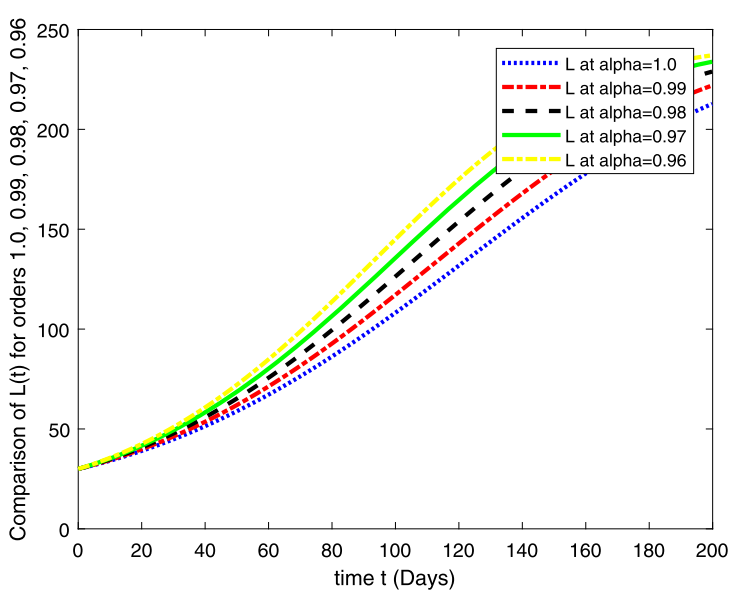

Figure 2 Light smokers for the orders 1.0, 0.99, 0.98, 0.97

Here the numerical scheme is applied to a particular case with the initial values $\mathcal{P}(0)=$ $100, \mathcal{L}=30, \mathcal{S}=10, \mathcal{Q}=20 . A=10.25, \beta=0.038, \delta=0.000274, \mu=0.0111, d=0.0019$, $\xi=0.021$, and $\gamma=0.006$.

In Fig. 1, we present a graphical representation of the simulation of the potential smokers $\mathcal{P}(t)$ in (43) for the fractional orders $1.0,0.99,0.98,0.97$. They increase up to 100 days and then decrease to a certain value. Comparing the results of fractional orders with integer orders, we see that the fractional-order results get closer to the classical results on values of the fractional orders closer to 1 . Figures 2 and 3 show the computational results for the light $\mathcal{L}$ and smokers $\mathcal{S}$.

In Fig. 4, a comparative analysis is given for the numerical simulations of the $\mathcal{Q}$ class of the smoking model (43). The final Fig. 5 shows a join solution of the model for order 1.

\section{Conclusions}

We considered a general class of fractional-order differential equations (FDEs). This area is still open for consideration of the sequential fractional differential equations, hybrid FDEs, 


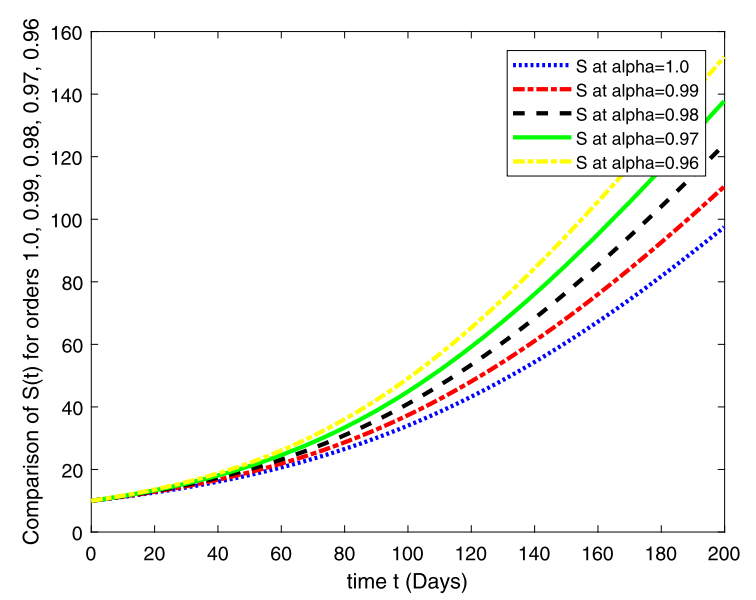

Figure 3 Smokers $\mathcal{S}$ for the orders 1.0, 0.99, 0.98, 0.97

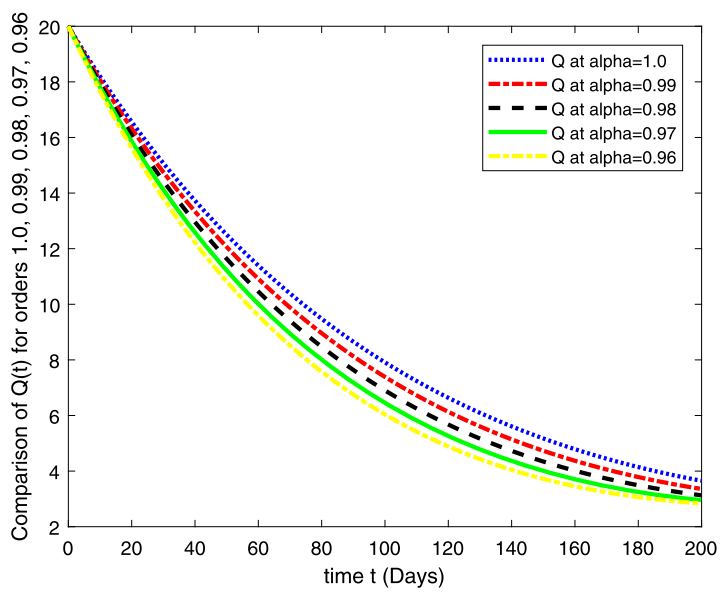

Figure 4 Quit smokers $S(t)$ for orders 1.0, 0.99, 0.98, 0.97

mixed fractional functional equations, and many more. The hybrid FDEs consist of two subclasses called the linear and quadratic differential equations. In this paper, we aimed to present a system of hybrid sequential FDEs with two different fractional operators, the Caputo and Atangana-Baleanu operators. We studied the existence and uniqueness of a solution. We have observed that some essential conditions are required for the existence of a solution of the fractional-order hybrid problem (1). There are two basic reasons for the importance. We have studied in the literature that the sequential hybrid class of FDS have not been considered for the presumed class for the existence of solution and stability analysis. Among them, one is a combination of the fractional derivative operators (the Caputo fractional differential operator and the Atangana-Baleanu fractional operator), whereas the second importance of the problem is the coupling of $n$ FDEs with complex boundary conditions. This can further motivate the readers to the combination of other operators and make more research problems for the initial and boundary conditions. The problem is converted into its equivalent integral form using Green's functions. Then the H-U stability is illustrated. Mathematical modeling of dynamical systems and their numerical simula- 


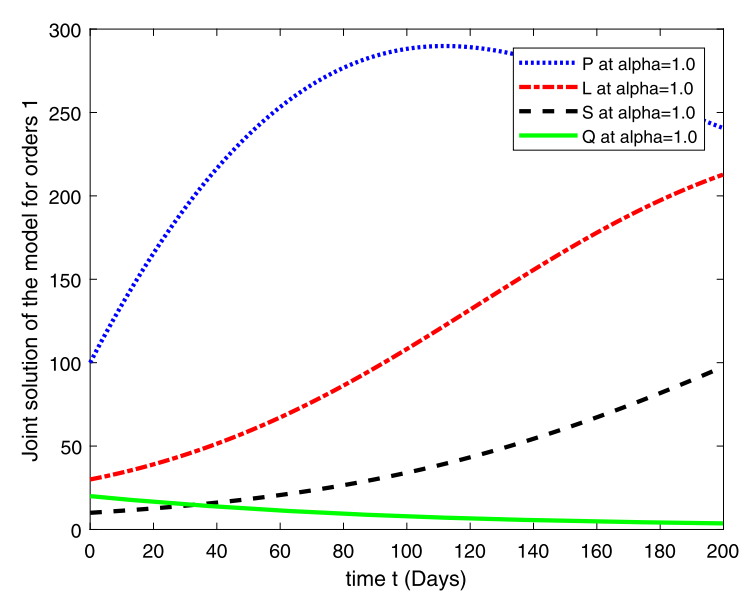

Figure 5 Joint solution for the model at 1.0

tions are considered as an application of the work. This aspect of the paper consists of a fractional-order smoking model studied for the numerical analysis. The numerical results are illustrated by some graphics based on our numerical scheme for model (43).

\section{Acknowledgements}

Princess Nourah bint Abdulrahman University Researchers Supporting Project number (PNURSP2022R8). Princess Nourah bint Abdulrahman University, Riyadh, Saudi Arabia.

Funding

Not applicable.

Availability of data and materials

Not applicable.

\section{Declarations}

Consent for publication

Not applicable.

\section{Competing interests}

The authors declare that they have no competing interests.

\section{Authors' contributions}

All the authors have equal contributions in this paper. All authors read and approved the final manuscript.

\section{Author details}

'Department of Mathematics and Sciences, Prince Sultan University, P.O. Box 66833, 11586 Riyadh, Saudi Arabia.

${ }^{2}$ Department of Mathematical Sciences, College of Science, Princess Nourah bint Abdulrahman University, P.O. Box 84428, Riyadh 11671, Saudi Arabia. ${ }^{3}$ Department of Medical Research, China Medical University, Taichung 40402, Taiwan.

${ }^{4}$ Department of Mathematics, Shaheed Benazir Bhutto University, Dir Upper 18000, Khybar Pakhtunkhwa, Pakistan.

\section{Publisher's Note}

Springer Nature remains neutral with regard to jurisdictional claims in published maps and institutional affiliations.

Received: 1 November 2021 Accepted: 7 January 2022 Published online: 02 February 2022

\section{References}

1. Deimling, K.: Nonlinear Functional Analysis. Springer, New York (1985)

2. Atangana, A., Araz, S.I: Mathematical model of Covid-19 spread in Turkey and South Africa: theory, methods and applications. Adv. Differ. Equ. 2020, 659 (2020)

3. Atangana, A., Baleanu, D.: New fractional derivatives with nonlocal and non-singular kernel: theory and application to heat transfer model. Therm. Sci. 20(2), 763-769 (2016) 
4. Atangana, A., Araz, S.I.: Nonlinear equations with global differential and integral operators: existence, uniqueness with application to epidemiology. Results Phys. 20, 103593 (2020)

5. Losada, J., Nieto, J.J.: Properties of a new fractional derivative without singular kernel. Prog. Fract. Differ. Appl. 1(2), 87-92 (2015)

6. Caputo, M., Fabrizio, M.: A new definition of fractional derivative without singular kernel. Prog. Fract. Differ. Appl. 1(2), $1-3(2015)$

7. Gorenflo, R., Mainardi, F.: Fractional calculus. In: Fractals and Fractional Calculus in Continuum Mechanics, pp. 223-276. Springer, Vienna (1997)

8. Atangana, A., Owolabi, K.M.: New numerical approach for fractional differential equations. Math. Model. Nat. Phenom. 13(1), 3 (2018)

9. Akgul, A., Modanli, M.: Crank-Nicholson difference method and reproducing kernel function for third order fractional differential equations in the sense of Atangana-Baleanu Caputo derivative. Chaos Solitons Fractals 127, 10-16 (2019)

10. Solis-Perez, J.E., Gomez-Aguilar, J.F., Atangana, A.: Novel numerical method for solving variable-order fractional differential equations with power, exponential and Mittag-Leffler laws. Chaos Solitons Fractals 114, 175-185 (2018)

11. Akinlar, M.A., Tchier, F., Inc, M.: Chaos control and solutions of fractional-order Malkus waterwheel model. Chaos Solitons Fractals 135, 109746 (2020)

12. Khan, H., Gomez-Aguilar, J.F., Khan, A., Khan, T.S.: Stability analysis for fractional order advection-reaction diffusion system. Phys. A, Stat. Mech. Appl. 521, 737-751 (2019)

13. Attia, N., Akgul, A., Seba, D., Nour, A.: An efficient numerical technique for a biological population model of fractional order. Chaos Solitons Fractals 141, 110349 (2020)

14. Atangana, A., Alqahtani, R.T.: New numerical method and application to Keller-Segel model with fractional order derivative. Chaos Solitons Fractals 116, 14-21 (2018)

15. Abdeljawad, T.: On Riemann and Caputo fractional differences. Comput. Math. Appl. 62(3), 1602-1611 (2011)

16. Abdeljawad, T., Fractional, B.D.: Differences and Integration by Parts. J. Comput. Math. 13(3) (2011)

17. Baitiche, Z., Guerbati, K., Benchohra, M., Zhou, Y.: Boundary value problems for hybrid Caputo fractional differential equations. Mathematics 7, 282 (2019)

18. Derbazi, C., Hammouche, H., Benchohra, M., Zhou, Y.: Fractional hybrid differential equations with three-point boundary hybrid conditions. Adv. Differ. Equ. 2019, 125 (2019)

19. Borai, M.M.E., Sayed, W.G.E., Badr, A.A., Tarek, A.: Initial value problem for stochastic hybrid Hadamard fractional differential equation. J. Adv. Math. 16, 8288-8296 (2019)

20. Hilal, K., Kajouni, A.: Boundary value problems for hybrid differential equations with fractional order. Adv. Differ. Equ. 2015, 183 (2015)

21. Ahmad, B., Ntouyas, S.K.: Initial-value problems for hybrid Hadamard fractional differential equations. Electron. J. Differ. Equ. 2014, 161 (2014)

22. Dhage, B.C.: Hybrid fixed point theory in partially ordered normed linear spaces and applications to fractional integral equations. Differ. Equ. Appl. 5, 155184 (2013)

23. Dhage, B.C., Dhage, S.B., Ntouyas, S.K.: Approximating solutions of nonlinear hybrid differential equations. Appl. Math. Lett. 34, 76-80 (2014)

24. Zhang, S.: The existence of a positive solution for a nonlinear fractional differential equation. J. Math. Anal. Appl. 252, 804-812 (2000)

25. Dhage, B.C., Lakshmikantham, V.: Basic results on hybrid differential equations. Nonlinear Anal. Hybrid Syst. 4, 414-424 (2010)

26. Dhage, B.: Quadratic perturbations of periodic boundary value problems of second order ordinary differential equations. Differ. Equ. Appl. 2, 465-486 (2010)

27. Dhage, B.: Periodic boundary value problems of first order Carathéodory and discontinuous differential equations. Nonlinear Funct. Anal. Appl. 13(2), 323-352 (2008)

28. Dhage, B.: Basic results in the theory of hybrid differential equations with mixed perturbations of second type. Funct. Differ. Equ. 19, 1-20 (2012)

29. Herzallah, M.A., Baleanu, D.: On fractional order hybrid differential equations. Abstr. Appl. Anal. 2014, Article ID 389386 (2014)

30. Mahmudov, N., Matar, M.M.: Existence of mild solution for hybrid differential equations with arbitrary fractional order. TWMS J. Pure Appl. Math. 8(2), 160-169 (2017)

31. Jafari, H., Baleanu, D., Khan, H., Khan, R.A., Khan, A.: Existence criterion for the solutions of fractional order $p$-Laplacian boundary value problems. Bound. Value Probl. 2015, 164 (2015)

32. Ahmad, B., Nieto, J.J.: Existence of solutions for impulsive anti-periodic boundary value problems of fractional order. Taiwan. J. Math. 15(3), 981-993 (2011)

33. Wang, G., Ahmad, B., Zhang, L., Nieto, J.J.: Comments on the concept of existence of solution for impulsive fractional differential equations. Commun. Nonlinear Sci. Numer. Simul. 19(3), 401-403 (2014)

34. Al-Sadi, W., Zhenyou, H., Alkhazzan, A.: Existence and stability of a positive solution for nonlinear hybrid fractional differential equations with singularity. J. Taibah Univ. Sci. 13(1), 951-960 (2019)

35. Khan, R.A., Gul, S., Jarad, F., Khan, H.: Existence results for a general class of sequential hybrid fractional differential equations. Adv. Differ. Equ. 2021(1), 284 (2021)

36. Aljoudi, S., Ahmad, B., Nieto, J.J., Alsaedi, A.: A coupled system of Hadamard type sequential fractional differential equations with coupled strip conditions. Chaos Solitons Fractals 91, 39-46 (2016)

37. Agarwal, P., Baleanu, D., Chen, Y., Momani, S., Machado, J.A.: Fractional calculus. In: Conference Proceedings ICFDA 2018, Amman, Jordan, July 16-18, 2018 (2018)

38. Rajchakit, G., Agarwal, P., Ramalingam, S.: Stability Analysis of Neural Networks (2021)

39. Agarwal, P., Baltaeva, U., Tariboon, J.: Solvability of the boundary-value problem for a third-order linear loaded differential equation with the Caputo fractional derivative. In: Special Functions and Analysis of Differential Equations, pp. 321-334 (2020)

40. Agarwal, P., Dragomir, S.S., Jleli, M., Samet, B. (eds.): Advances in Mathematical Inequalities and Applications Springer, Singapore (2018) 
41. Ruzhansky, M., Cho, Y.J., Agarwal, P., Area, I. (eds.): Advances in Real and Complex Analysis with Applications Springer, Singapore (2017)

42. Salahshour, S., Ahmadian, A., Senu, N., Baleanu, D., Agarwal, P.: On analytical solutions of the fractional differential equation with uncertainty: application to the Basset problem. Entropy 17(2), 885-902 (2015)

43. Tariboon, J., Ntouyas, S.K., Agarwal, P.: New concepts of fractional quantum calculus and applications to impulsive fractional $q$-difference equations. Adv. Differ. Equ. 2015(1), 18 (2015)

44. Morales-Delgado, V.F., Gómez-Aguilar, J.F., Saad, K.M., Khan, M.A., Agarwal, P.: Analytic solution for oxygen diffusion from capillary to tissues involving external force effects: a fractional calculus approach. Phys. A, Stat. Mech. Appl. 523, 48-65 (2019)

45. Thabet, S.T., Etemad, S., Rezapour, S.: On a coupled Caputo conformable system of pantograph problems. Turk. J. Math. 45, 496-519 (2021)

46. Baleanu, D., Etemad, S., Rezapour, S.: On a fractional hybrid integro-differential equation with mixed hybrid integral boundary value conditions by using three operators. Alex. Eng. J. 59(5), 3019-3027 (2020)

47. Baleanu, D., Etemad, S., Rezapour, S.: A hybrid Caputo fractional modeling for thermostat with hybrid boundary value conditions. Bound. Value Probl. 2020(1), 64 (2020)

48. Mohammadi, H., Kumar, S., Rezapour, S., Etemad, S.: A theoretical study of the Caputo-Fabrizio fractional modeling for hearing loss due to Mumps virus with optimal control. Chaos Solitons Fractals 144, 110668 (2021)

49. Matar, M.M., Abbas, M.I., Alzabut, J., Kaabar, M.K., Etemad, S., Rezapour, S.: Investigation of the $p$-Laplacian nonperiodic nonlinear boundary value problem via generalized Caputo fractional derivatives. Adv. Differ. Equ. 2021(1), 68 (2021)

50. Alizadeh, S., Baleanu, D., Rezapour, S.: Analyzing transient response of the parallel RCL circuit by using the Caputo-Fabrizio fractional derivative. Adv. Differ. Equ. 2020(1), 55 (2020)

51. Baleanu, D., Rezapour, S., Saberpour, Z:: On fractional integro-differential inclusions via the extended fractional Caputo-Fabrizio derivation. Bound. Value Probl.. 2019(1), 79 (2019)

52. Baleanu, D., Etemad, S., Pourrazi, S., Rezapour, S.: On the new fractional hybrid boundary value problems with three-point integral hybrid conditions. Adv. Differ. Equ.. 2019(1), 473 (2019)

53. Aydogan, M.S., Baleanu, D., Mousalou, A., Rezapour, S.: On high order fractional integro-differential equations including the Caputo-Fabrizio derivative. Bound. Value Probl. 2018(1), 90 (2018)

54. Baleanu, D., Mohammadi, H., Rezapour, S.: Analysis of the model of HIV-1 infection of CD4+ CD4+ T-cell with a new approach of fractional derivative. Adv. Differ. Equ. 2020(1), 71 (2020)

55. Caputo, M., Fabrizio, M.: A new definition of fractional derivative without singular kernel. Prog. Fract. Differ. Appl. 1(2), $1-3(2015)$

56. Losada, J., Nieto, J..: Properties of a new fractional derivative without singular kernel. Prog. Fract. Differ. Appl. 1(2), 87-92 (2015)

57. Atangana, A., Baleanu, D.: New fractional derivatives with nonlocal and non-singular kernel: theory and application to heat transfer model. Therm. Sci. 20(2), 763-769 (2016)

58. Jarad, F., Abdeljawad, T., Hammouch, Z:: On a class of ordinary differential equations in the frame of Atangana-Baleanu fractional derivative. Chaos Solitons Fractals 117, 16-20 (2018)

59. Alzaid, S.S., Alkahtani, B.S.: Asymptotic analysis of a giving up smoking model with relapse and harmonic mean type incidence rate. Results Phys. 28, 104437 (2021)

\section{Submit your manuscript to a SpringerOpen ${ }^{\circ}$ journal and benefit from:}

- Convenient online submission

- Rigorous peer review

- Open access: articles freely available online

- High visibility within the field

- Retaining the copyright to your article

Submit your next manuscript at $\gg$ springeropen.com 